\title{
Lathe Chuck Wrench Anti-crash Alarm System
}

\author{
Junyan Chen', Kaiyu Tang ${ }^{2}$, Yao Ma ${ }^{1}$, Yan Li ${ }^{3}$, Ning Wang', and Zhibin Wang \\ ${ }^{1}$ College of Mechanical and Electrical Engineering, Shenyang Aerospace Shenyang Aerospace University, \\ Shenyang 110136, China; \\ ${ }^{2}$ Engineering Training Center, Shenyang Aerospace University, Shenyang 110136, China. \\ ${ }^{3}$ College of Innovation and Entrepreneurship, Shenyang Aerospace University, Shenyang 110136, China
}

Keywords: card wrench, KY18 metal sensor, microcomputer, WAV player, SD card read.

\begin{abstract}
This paper expounds the necessity of setting up safety protection device for ordinary lathe, and further analyzes the subjective and objective factors of safety accidents caused by chuck wrench. This device detects lathe chuck by metal touch sensor. When the module comes into contact with the three-jaw chuck, the signal is transmitted to the Arduino single-chip microcomputer to alarm. The work principle of the design and the structure of the safety protection system are described in detail.
\end{abstract}

\section{Introduction}

In the process of lathe work, accidents occur frequently for various reasons, which have a great impact on the mechanical processing industry. Generally speaking, inexperienced operators or college students who have just entered the internship factory are the most vulnerable groups. Due to the lack of practical experience, the cognition of lathe processing is in the beginning stage. Therefore, there is a frequent occurrence of chuck wrench to fly out of the safety accident. Every occurrence of safety accidents will have a negative impact on enterprises and schools, and also cause physical and psychological damage to the operators [3].This will both damage the device and create a psychological shadow over the operator. In order to avoid the occurrence of tragedy, it is necessary to design reasonable anti - crash alarm.

\section{Principle}

Figure 1 for Proteus simulation software for single-chip microcomputer, when KY18 metal sensor (FIG. Instead of using photosensitive resistance) ventilation with high electricity at ordinary times, namely KY18 metal sensor give no detected the light source of the lathe chuck, alarm buzzer will not occur. When the light source of the KY18 metal sensor is detected to the lathe chuck, the single chip will drive the buzzer to sound the alarm.[5] during normal operation, the device will not produce any interference sound, and only alarm when the chuck wrench is not removed.

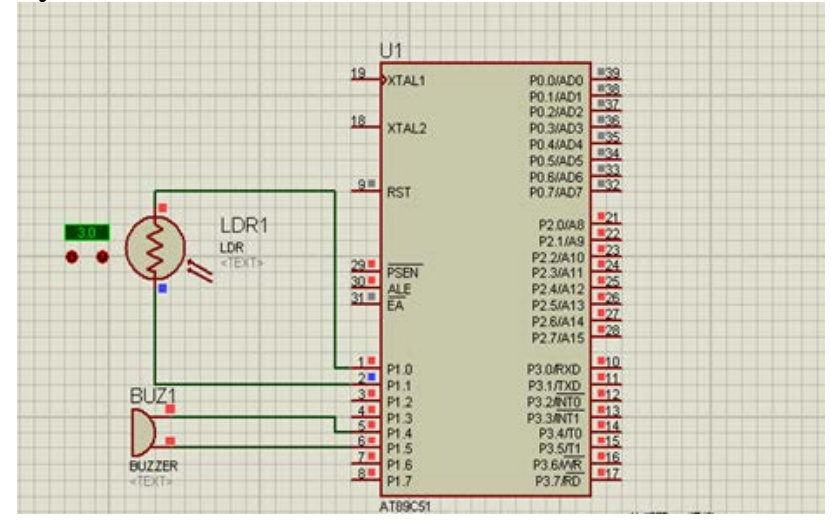

Fig. 1 The simulation of Proteus software for single chip microcomputer 
Design. The control part of the lathe chuck wrench anti-impact alarm is mainly composed of three parts:

1) Arduino R3 improvement board 1, R3 I/O expansion board 1.

2) SD card reading module 1 , speaker 1.

3) KY18 metal sensor 1.

By design, lathe chuck wrench injury prevention alarm system, need a total of $15 \mathrm{I} / \mathrm{O}$ port, through the network to buy the Arduino a R3 SCM, but because of the need to more pins can output PWM wave, on the Arduino R3 microcontroller, found that only 8 pin mouth can output PWM signals, and so on the basis of the Arduino R3 microcontroller, then bought a R3 development board, through the expansion board, expanded the MCU I/O port, eventually be able to complete communication between modules and modules.

Because the alarm function of this device is to make a human voice, to improve the user's vigilance, through discussion, we decided to use the existing SD card reading module on the Internet. The SPI communication interface of the SD card enables it to read and write data through the SPI channel. From the perspective of application, the advantage of using SPI interface is that many SCM internal SPI controller not only brings convenience to development, but also reduces development cost. However, it also has some disadvantages, such as losing the performance advantage of SD card, to solve this problem, it is necessary to use SD mode, because it provides more bus data bandwidth. The selection of the SPI interface is done when the first command is written to it at the initial time. The following is the driver method of SD card, which can only be read and write in a simple sector.

After a read SD card module, we also need a player, first by the written information within the SD card read out, again will read out the information broadcast, we ended up in choosing WAV player module, it is important to note that the WAV player module, will need the following file is 16 bit, mono, $22.1 \mathrm{kHz}$ sampling rate under WAV audio file format, This is because the audio CD is used in the dual tone even more sound way, and as high as $44.1 \mathrm{KHz}$ sampling rate, high frequency, will increase the volume of a music file, at the same time, the tone and the word was with more sound way without too much difference in terms of "remind", taking low frequency under the condition of, also won't lead to remind sound distortion, ultimately achieve the purpose of the is: through the small file, a little bit more simple procedures, can complete the lathe chuck wrench injury prevention alarm system design. PS: If music files are multi-channel and $44.1 \mathrm{KHz}$, the properties of music files and the sampling rate can be reduced to $22.1 \mathrm{KHz}$ through the online format chemical factory.

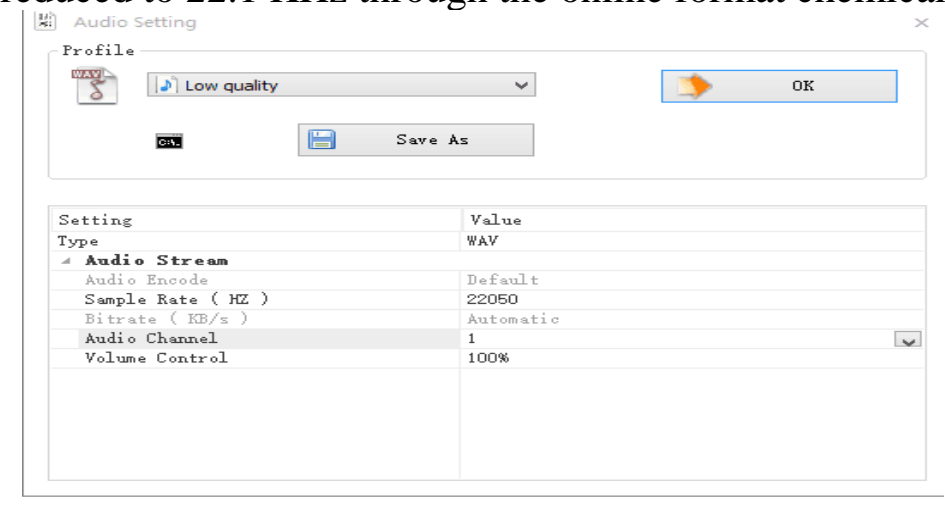

Fig.2 A screenshot of a chemical factory

As shown in the figure above, the program block diagram of the overall lathe chuck wrench anti-collision warning device is shown in the figure above. After the power of the single-chip computer, the reading and writing of the SD card is first performed. If the presence of music files in the SD card does not meet the 16 bit, mono, $22.1 \mathrm{kHz}$ sampling rate under the following the wav format audio files, through the Arduino UNO R3 serial port monitor as you can see, can't work for SD card read and write, will be an error. 


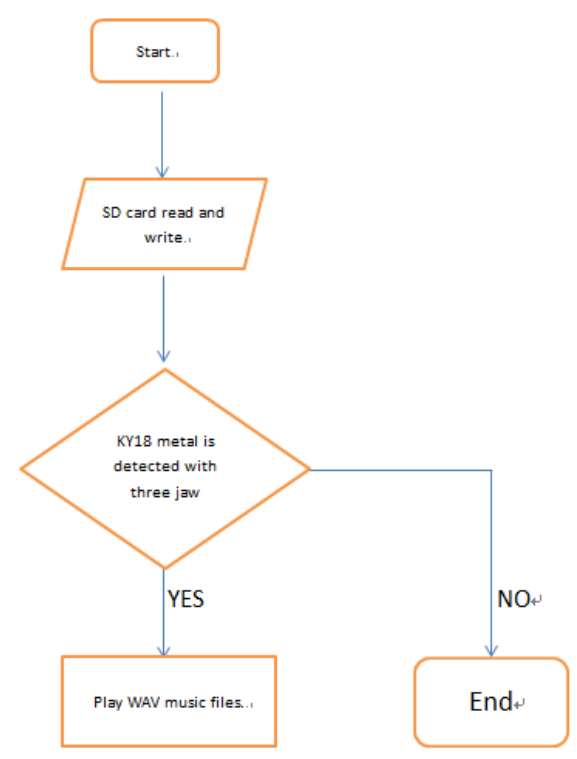

Fig.3 Program block diagram

In the SD card read, after the completion of work process will be in accordance with the program block diagram in figure 8, to read KY18 metal sensor, in order to make SCM know, when to detect three jaw chuck, and play the recorded WAV files in advance, so you need to connect the I/O KY metal sensor mouth into the OUTPUT, when the new metal sensor to detect metal (three jaw chuck), read by the program, it will give the single-chip digital signal "1", reflect is the digital signal in the single chip microcomputer, is like a judge in a program flowchart is "KY18 metal sensor to detect three jaw chuck?" In order to be able to timely reflect the signal, in the process of writing programs, through two ways to achieve this control method, the first is through the statement, the second is whether to MCU by external interrupt transmission detected three jaw chuck.

This time is mainly through the method of external interrupt detection.

After the KY18 metal sensor detects the three-jaw chuck, the WAV player starts and stops with the program implementation.

Burn the previously written program to the Arduino R3 expansion board. When the RX and TX lights flicker, the program burns successfully. After successful installation of the alarm, the lathe chuck is detected by metal sensors in FIG. 2 and 3. When the three jaw chuck is detected, the signal is transmitted to the Arduino R3 controller. By amplifying circuits inside the speaker and the number of electric, electric conversion, the point of the signal into sound signal, in under the action of the speaker, will have been issued a "notice, chuck wrench did not take off!"The sound of alarm can remind the operator to remove the lathe chuck wrench, so as to avoid the chuck wrench flying out of the three jaw chuck, causing the accident [4].

\section{Connection}

1) Check the IO expansion board on the improved motherboard.

2) Connect the Micro SD card module to the IO expansion board with the dupont line and plug in the Micro SD card.

3) Connect the KY18 metal sensor module to the IO expansion board.

4) Connect the wav player module to the IO expansion board and plug in the speaker.

5) Since there is no library file for the WAV player we need in the library file of the Arduino MCU, we need to advance to the Arduino Chinese community to download the library files of the WAV player. Finally will download good WaveHC library file copy to your Arduino IDE library file directory, in after the success of the copy, you can call WAV library file, in this way, can more convenient to realize play WAV music files.

6) Connect the motherboard to the computer with the USB cable, Arduino microcontroller need more than $5 \mathrm{~V}$ or $5 \mathrm{~V}$ voltage, we don't have to worry about, the voltage is too large, connected to the 
Arduino microcontroller, the issues that led to the Arduino microcontroller will be burn out in a lot of online purchase in the Arduino microcontroller, its power supply interface (by ordinary android line) and AVR connection between the minimum control system (Arduino) have a zener diode, it can be power input to the output to a minimum voltage stability of the control system in $3.3 \mathrm{~V}$, so the Arduino directly connected to the computer, is don't have to worry about this problem.

\section{Conclusion}

The design of some existing lathe safety production equipment is realized by changing the electrical control in the lathe. The cost of transformation is very difficult to popularize widely. Incontrast, the device is relatively simple, safe and reliable, light weight, convenient installation and low price, suitable for large-scale promotion in universities, higher vocational colleges and vocational schools.

\section{Acknowledgments}

This work is partly supported by College students Creation and Career Training Project of Shenyang Aerospace University, NO.110317059 and the National College Students Creation and Career Training Project in years of 2017, No.201710143000175.

\section{References}

[1] Du xiaoling, Yang zuo, zouyongbing. The necessity and use effect of general lathes for the interlock safety device of chuck, and the journal of chongqing industrial college, 2005.02.

[2] Gao Dechong. Safety device in the lathe chuck wrench improved design of the application/technology BBS.

[3] Cheng liming. Design and application of safety protection device for lathe chuck wrench and application/China modern education equipment /2009.11.

[4] Zhang xin and pan wenlong. Safety protection of lathe chuck wrench. Design and application of protective device/silicon valley /2013.0.

[5] Kadowaki,Yoshalsupu/DDEVELOPMENTOF CHUCKINGCONDITION THREE-AW SCROLLCHUCK/SourceBulingth.SME,v29,n248.p625-631,Fb1986

SENSORFOR 